\title{
The development of a new complexation technique of hydrocortisone acetate with 2-hydroxypropyl- $\beta$ - cyclodextrin: preparation and characterization
}

\begin{abstract}
In the present study, an inclusion complex composed of hydrocortisone acetate (HA) and 2-hydroxypropyl- $\beta$-cyclodextrin (HP $\beta C D$ ) was prepared according phases solubility diagram of Higuchi \& Connors and by a method described by Filipović-Grcić J et al..' with minor modifications to improve the HA water solubility. This new method improved percentage of inclusion reaching the maximum percentage of inclusion $(91.52 \pm 0.92 \%)$ using $5.75 \mathrm{mM} \mathrm{HA}$ and $135 \mathrm{mM} \mathrm{HP} \beta \mathrm{CD}$. This percentage of inclusion was higher than that found by Filipović-Grcić J et al.. ${ }^{1}$ which was $25 \%$. The complex HA/HP $\beta C D$ was characterized by $1 \mathrm{H}-\mathrm{NMR}$ through chemical shifts in $1 \mathrm{H}$ NMR spectra after the inclusion of HA into the HP $\beta C D$ cavity, especially H-3, H-5 and H-6 protons was observed to the formation of molecular inclusion complex. Other technique used to characterize the inclusion complex was FT-IR, which showed in the FT-IR spectra of inclusion complex no features bands similar to pure HA molecule; the spectrum is very similar to the HP $\beta C D$. The molecular modeling studies results are in agreement with the experimental data that estimates a HA/ $\mathrm{HP} \beta C D$ molar ratio of $1: 1$. The calculated apparent stability constant $(K \mathrm{~S})$ was $484 \mathrm{M}-1$. The results indicate that the HA/HP $\beta C D$ inclusion complex is more water soluble than HA crystalline powder (pure), the AH solubility was increased on the order of 64 times.
\end{abstract}

Keywords: 2-hydroxypropyl- $\beta$-cyclodextrin; Hydrocortisone acetate; Inclusion complex; FT-IR; 1H-NMR and modeling molecular
Volume 7 Issue I - 2018

\author{
Márcio Robert Mattos da Silva,' Elisabete \\ Pereira dos Santos,' Rita de Cássia da Silva \\ Assunção Barros,' Sheila Garcia,' Magaly \\ Girão Albuquerque,' Jackeline da Silva \\ Coelho Oliveira, ${ }^{2}$ Márcia Soares Sader ${ }^{3}$ \\ 'Department of Medicines, Federal University of Rio of Janeiro, \\ Brazil \\ ${ }^{2}$ Department of Organic Chemistry, Federal University of Rio \\ of Janeiro, Brazil \\ ${ }^{3}$ Metallurgical and Materials Engineering Program, Federal \\ University of Rio of Janeiro, Brazil
}

\begin{abstract}
Correspondence: Márcio Robert Mattos da Silva, Department of Medicines, Faculty of Pharmacy, Federal University of Rio of Janeiro, University Pharmacy, Center of Health Sciences, Block K, room 50, 2o floor, RJ, Zip code: 2 194I-590, Brazil, Email mattos25@zipmail.com.br
\end{abstract}

Received: December 27, 2017 | Published: January 17, 2018
Abbreviations: $\mathrm{HP} \beta \mathrm{CD}$, 2-hydroxypropyl- $\beta$-Cyclodextrin; HA, hydrocortisone acetate; FT-IR, fourier transform infrared spectroscopy; 1H-NMR, 1H nuclear magnetic resonance; CDs, cyclodextrins; Ks: apparent stability constant; RMS, root mean squares

\section{Introduction}

The Hydrocortisone acetate (Figure 1) is a white crystalline powder; it melts at about $220^{\circ} \mathrm{C}$ with decomposition and is practically insoluble in water $1 \mathrm{mg} / 100 \mathrm{~mL} .^{2}$ One method to overcome the poorly water solubility of HA is the use of cyclodextrins (CDs), which have been extensively studied. ${ }^{3}$ CDs have been extensively used as complexing agents to improve the solubility of a variety of poorly soluble drugs. ${ }^{4} \mathrm{CDS}$ are regarded by pharmaceutical scientists as a new group of pharmaceutical excipients. ${ }^{5}$

CDs are macrocyclic torus-shaped molecules formed by D $(+)$ - glucopyranose units. CDs size and shape are correlated to the type and number of $(1,4)$ linkages between those units and contain a somewhat lipophilic central cavity and a hydrophilic outer surface. CDs are capable of forming inclusion complexes with various types of organic compounds with improve solubility or other physicochemical properties. ${ }^{3}$ Due to the unique truncated cone structure of CDs, it can accommodate hydrophobic guest molecules inside the cavity ${ }^{6}$ (Figure 2A). The size and geometry of the molecule to be included in the $\mathrm{CD}$ must be adequate for the size and shape of the non-polar cavity. In general, organic compounds with molecular weights between 200 and $800 \mathrm{~g} / \mathrm{mol}$ can be included in one of the three cavities formed by the $(\alpha, \beta$ and $\gamma)$ CDs. Inclusion complexes may occur in solid or in solution. ${ }^{7}$ The organic compounds enter partly or entirely into the relatively hydrophobic cavity of CDs simultaneously expelling the few high-energy water molecules from inside. ${ }^{7}$ The driving forces for complex formation are van der Waals, hydrophobic or hydrogen bond interactions. ${ }^{9,10}$

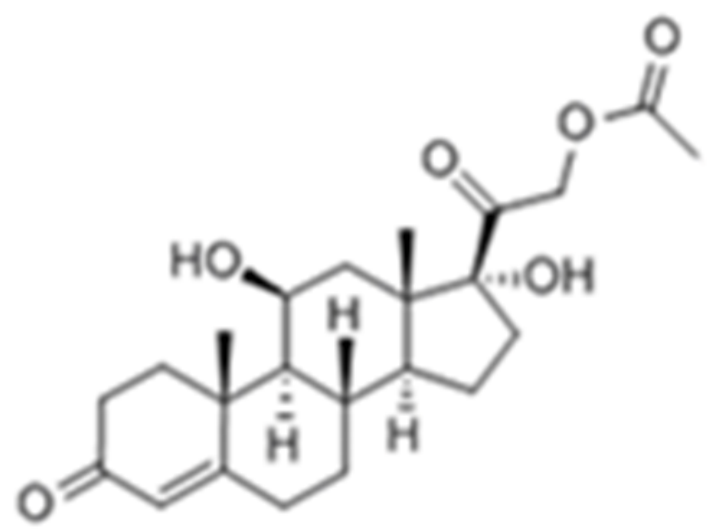

Figure I Hydrocortisone acetate.

The 2-hydroxypropyl- $\beta$-cyclodextrin $(\mathrm{HP} \beta \mathrm{CD}$ ) is a chemical modified $\mathrm{CD}$, where the group 2-hydroxypropyl replaces hydrogen atoms of the free groups (Figure $2 \mathrm{~B}$ ). ${ }^{3}$ The HP $\beta C D$ which is known for its good aqueous solubility and low toxicity ${ }^{11}$ and the cost is within the range of common surfactants. ${ }^{12}$

There are few reports on the inclusion complex of HA and HP $\beta C D$ in the scientific literature. Previous studies have discovered that the water solubility of HA can be largely enhanced by complexation with $\mathrm{HP} \beta \mathrm{CD} .{ }^{1}$ In that work the inclusion complex of HA with HP $\beta \mathrm{CD}$ 
were performed as the following technique; HA and HP $\beta C D$ (1:1 ratio molar) were dissolved separately in ethanol and purified water, respectively. The solutions were mixed together, sonicated for 15 minutes to produce a clear solution and subjected to spray-drying, achieving $25 \%$ inclusion of $\mathrm{AH}$.

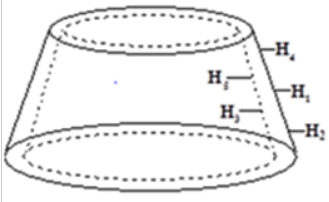

(2a)

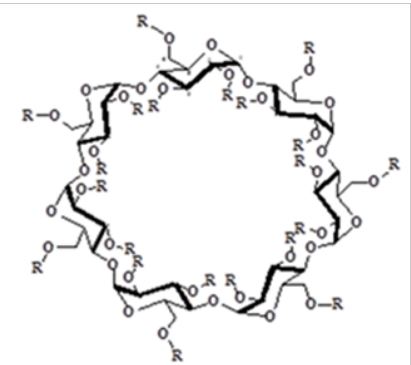

(2b)
Figure 2 (A) Schematic representation of the truncated cone structure of the CDs. (b): $\mathrm{HP} \beta C D\left(\mathrm{R}=\mathrm{CH}_{2} \mathrm{CHOHCH}_{3}\right)$

The aim of the present work was development a new technique of complexation of HA with HP $\beta C D$ to increase the inclusion percentage and the solubility of HA molecule. The best correlation of HA inclusion in $\mathrm{HP} \beta \mathrm{CD}$, stoichiometry, apparent stability constant $(K s)$ and quantification of yield of the freeze-drying inclusion complex were determined. The inclusion complex was analyzed by different analytical techniques including Fourier transform-infrared spectroscopy (FT-IR) and 1H Nuclear magnetic resonance (1H-NMR). Molecular modeling studies were performed to investigate the mode of binding of $\mathrm{AH}$ in $\mathrm{HP} \beta \mathrm{CD}$.

\section{Material and methods}

\section{Reagents}

2-hydroxypropyl- $\beta$-cyclodextrin was supplied by Cargill (E.U.A), hydrocortisone acetate standard and deuterated dimethyl sulfoxide (DMSO-d6) were purchase from (Sigma $\left.{ }^{\circledR}\right)$. All solvents used were of analytical reagent grade.

\section{Apparatus}

Analytical balance Mettler-Toledo AG-204, destilator Quimis, $\mathrm{pH}$ meter Digimed DME-CV-4, UV-Visible spectrophotometer Shimadzu UV-2401-PC, Fourier Transform Infrared Spectrometer Shimadzu FT-IR-8300, freeze dryer Labconco 603886, thermostatic bath Thornton T14 and spectrometer of nuclear magnetic resonance Bruker-Spectrospin DRX 400MHz.

\section{Preparation of the inclusion complex AH/HP $\beta C D$ and quantification of the inclusion percentage}

In this work, we have prepared an inclusion complex of HA with HP $\beta C D$ according phases solubility diagram of Higuchi \& Connors and a method described by Filipović-Grcić $\mathrm{J}$ et al.. ${ }^{1}$ with minor modifications, changing the solvent ethanol by water and using freeze-drying as a drying technique. Were accurately weighed $50,0 \mathrm{mg}$ of HA in a conical flask of $125 \mathrm{~mL}$ and in each flask was added slowly $\mathrm{HP} \beta C D$ aqueous solution of increasing concentration $(20,50,70,75$, $80,85$ and $135 \mathrm{mM})$. All samples had the final fixed concentration of HA $2 \mathrm{mg} / \mathrm{mL}$. The total volume inside the flasks was $25,0 \mathrm{~mL}$. The flasks were sealed and protect from the light with aluminum foil. The mixture was stirred at $37^{\circ} \mathrm{C}$ under water bath during 1 hour. This time and temperature were determined after the development of other conditions. After, the samples were filtered (Whatman ${ }^{\circledR} n^{\circ} 41$ ).
Clear solutions were suitably diluted in ethanol with theoretical final concentration of HA $20 \mathrm{mg} / \mathrm{mL}$ and analyzed by UV-vis spectrophotometry, measuring the absorbance at $241,5 \mathrm{~nm}$ wavelength (REF). The percentage of inclusion of HA were determined from a calibration curve of HA standard $(y=0,0405 x+0,0256 ; r=0,9941)$. The residue of filtrate with the highest percentage of inclusion of $\mathrm{AH}$ in $\mathrm{HP} \beta C D$ was freeze-dried and the resulting powders were directly used for quantification of yield and characterization of the inclusion complex.

\section{Determination of stoichiometry, apparent stability constant and increase of solubility}

The stoichiometry of HA/HP $\beta C D$ complex in solution was determined by the solubility technique. According to Higuchi and Connors's theory, ${ }^{13}$ if the saturation concentration of HA versus the concentration of HP $\beta C D$ in solutions yield a linear relationship this may be attributed to the formation of a 1:1 inclusion complex between $\mathrm{HA}$ and $\mathrm{HP} \beta \mathrm{CD} .{ }^{8}$

The apparent stability constant $(K s)$ of the inclusion complex formed was calculated from the straight-line portion of the phases solubility diagram using the equation (1), where $\mathrm{S} 0$ is the $\mathrm{AH}$ solubility in the absence of HP $\beta C D$ (intercept) ${ }^{14}$

Equation 1: $K \mathrm{~S}=$ Slope $/ \mathrm{S} 0$ (1 - slope)

The increased solubility (n) of HA was calculated as the ratio between the maximum solubility of the complex (Smax) and the solubility of HA in the absence of HP $\beta C D$ (SO).

\section{Quantification of yield and characterization of the freeze-dried inclusion complex}

The yield of the freeze-drying inclusion complex was calculated. The inclusion complex of HA/HPBCD was characterized by the following techniques.

\section{Fourier transform infrared spectroscopy (FT-IR)}

Fourier transform infrared spectroscopy (FT-IR) spectra of HA, $\mathrm{HP} \beta C D$, the physical mixture and the inclusion complex were collected from 4000 to $500 \mathrm{~cm}^{-1}$ using $\mathrm{KBr}$ disk technique. The samples were ground with spectroscopic grade potassium bromide $(\mathrm{KBr})$ powder during 5 minutes and then pressed into pellets $(3 \mathrm{mg}$ of sample per $300 \mathrm{mg}$ dry $\mathrm{KBr}$ ). A blank disk was used as background.

\section{H Nuclear magnetic resonance (I H-NMR)}

$1 \mathrm{H}$ Nuclear magnetic resonance $(1 \mathrm{H}-\mathrm{NMR})$ spectra of the physical mixture and the inclusion complex were dissolved in DMSO-d6 and analyzed at $400 \mathrm{MHz}$. Chemical shifts were expressed per million.

\section{Molecular modeling studies}

Molecular structure of the inclusion complex that showed the higher percentage was established using computational modeling technology HyperChem 7.5 program (Hyperchem 2002) to visually illustrate the formation of $\mathrm{AH} / \mathrm{HP} \beta \mathrm{CD}$ complex. The $\mathrm{HPbCD}$ and HA structures were submitted to the geometry optimization process simulating each molecule in vacuum..$^{15}$ and without any geometric restriction, using the molecular mechanics forcefield. ${ }^{16} \mathrm{MM}+$ of the HyperChem program, up to the lower gradient norm at 10-2 kcal mol$1 \AA-1$. The electrostatic term was calculated using the bond dipoles included in set parameter from the $\mathrm{MM}+$ force field. The inclusion complex was obtained by manually docking of the optimized HPbCD and $\mathrm{AH}$ structures and subjected to the same geometry optimization process. 


\section{Results and discussion}

\section{Quantification of inclusion complex}

Table 1 show the percentage values of the inclusion of HA in $\mathrm{HPbCD}$ determined by UV spectrophotometry. There was an increase in the percentage of inclusion of the $\mathrm{AH}$ with the increase of the concentration of $\mathrm{HPbCD}$, reaching the maximum inclusion limit of $91.52 \pm 0.92 \%$ when using a concentration ratio of $5.75 \mathrm{mM}$ of $\mathrm{HA}$ and $135 \mathrm{mM}$ of $\mathrm{HPbCD}$. This percentage was higher than that obtained by Filipović-Grcić J et al. ${ }^{1}$ which was $25 \%$.

Table I Percentage of inclusion of HA in HP $\beta C D$ containing HA at constant concentration and $\mathrm{HP} \beta C D$ at increasing concentration

\begin{tabular}{lll}
\hline HA $(\mathbf{m M})$ & HPßCD $(\mathbf{m M})$ & \% Inclusion complex \\
\hline 4,94 & 20 & $18,21 \pm 0,82$ \\
5,08 & 50 & $41,72 \pm 0,96$ \\
4,94 & 70 & $52,30 \pm 1,03$ \\
4,94 & 75 & $56,92 \pm 1,54$ \\
4,98 & 80 & $59,24 \pm 1,32$ \\
4,94 & 85 & $65,22 \pm 0,64$ \\
5,75 & 135 & $91,52 \pm 0,92$ \\
\hline
\end{tabular}

\section{Determination of stoichiometry, apparent stability constant and increase of solubility}

The stoichiometry of the inclusion complex HA/HP $\beta C D$ was determined by the solubility technique. As showed in Figure 3, a linear relationship was obtained between the amount of HA solubilized and the increasing of concentration of $\mathrm{HP} \beta C D$ in solution, which was classified as a classic AL-type, suggesting to the formation of inclusion complex in 1:1 molar ratio of $\mathrm{HA} / \mathrm{HP} \beta \mathrm{CD}$, in agreement with the previous study from Filipović-Grcić $\mathrm{J}$ et al. ${ }^{1}$. The regression equation was as follows: $\mathrm{y}=0,0769+0,0359 \mathrm{x}(\mathrm{r}=0,9979)$.

The apparent stability constant of the complex in the 1:1 molar ratio $K S(1: 1)$ was calculated from the solubility diagram, considering the angular coefficient (slope $=0.0359$ ) and HA solubility in the absence of $\mathrm{HP} \beta \mathrm{CD}(\mathrm{S} 0=0.0769 \mathrm{mM})$. The calculated $K S(1: 1)$ was $484 \mathrm{M}-1$, which is within the range considered suitable for the formation of stable inclusion complexes $100-5000 \mathrm{M}-1 .{ }^{17}$. The $K S(1: 1)$ calculated by Filipović-Grcić $\mathrm{J}$ et al.. ${ }^{12}$ was equal to $466 \mathrm{M}^{-1}$. The relation between $\mathrm{Smax}=4.9338 \mathrm{mM}$ and $\mathrm{So}=0.0769 \mathrm{mM}$, demonstrated that the increase of HA solubility was on the order of 64 times.

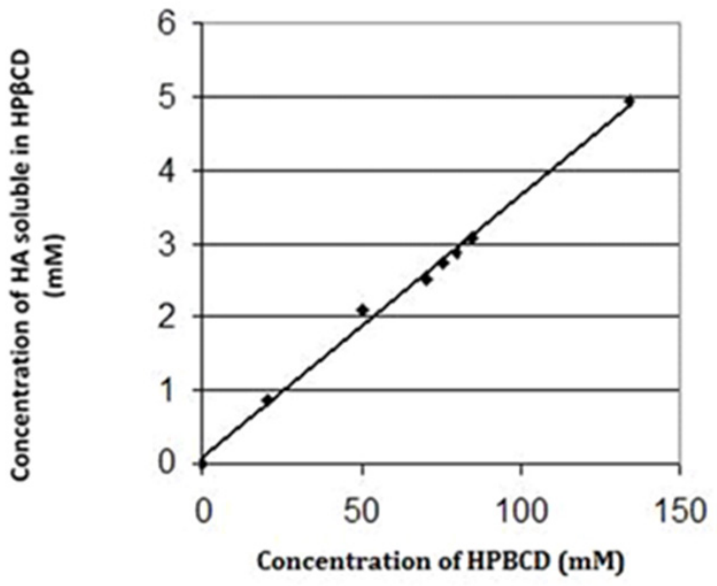

Figure 3 Hydrocortisone-HP $\beta C D$ phase solubility diagram in aqueous solution at $37^{\circ} \mathrm{C}$.

\section{Quantification of yield and characterization of the freeze-dried inclusion complex}

The freeze-dried inclusion complex presented as amorphous white powder. The yield of the inclusion process calculated was $77.4 \pm 0.85 \%$.

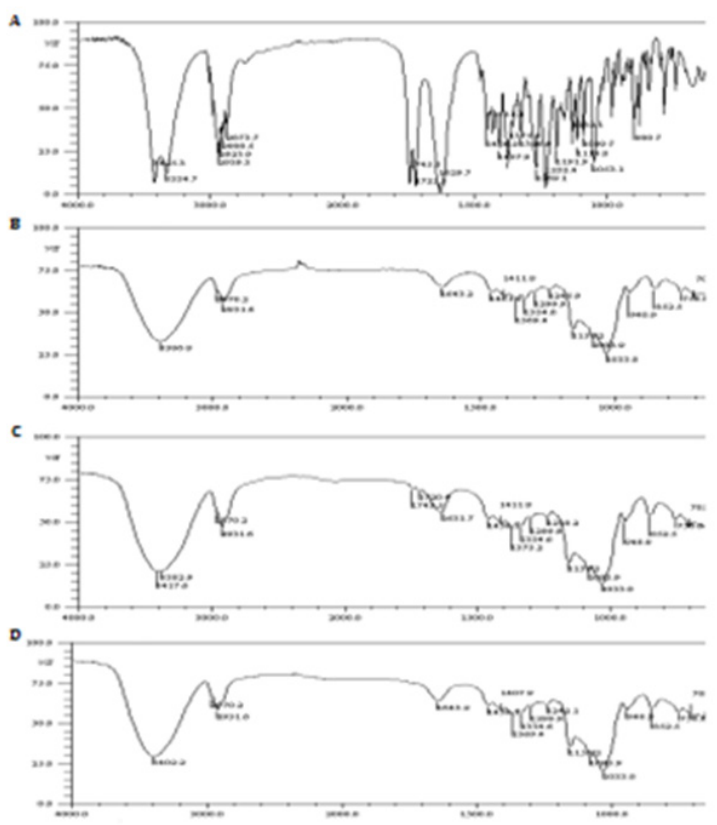

Figure 4 FT-IR spectra of HA (A), HPßCD (B), the physical mixture $(C)$ and the freeze-dried HA/HP $\beta C D$ inclusion complex (D).

\section{Characterization of inclusion complex}

Fourier transform infrared spectroscopy analysis: FT-IR is a useful technique used to confirm the formation of an inclusion complex. ${ }^{8}$ The FT-IR spectra of HA (A), HP $\beta C D(B)$, physical mixture and (C) and inclusion complex (D) are presented in the Figure 4. The FT-IR spectrum of HA showed bands features of HA at $3425,3 \mathrm{~cm}^{-1}$ and $3334,7 \mathrm{~cm}^{-1}$, both for $\mathrm{O}-\mathrm{H}$ stretching vibrations from the hydroxyl groups of the secondary and tertiary alcohols. The bands presented in the region between $3000 \mathrm{~cm}^{-1}$ and $2850 \mathrm{~cm}^{-1}$ are attributed stretching vibrations from the many types of bonds C-H. At $1745,5 \mathrm{~cm}^{-1}$, $1722,3 \mathrm{~cm}^{-1}$ and $1629,7 \mathrm{~cm}^{-1}$ the bands are stretching vibrations of bonds $\mathrm{C}=\mathrm{O}$ from carbonyl of acetate, ketone $\alpha$-hidroxylated and ketone $\alpha, \beta$-unsaturated, respectively. At $1232,4 \mathrm{~cm}^{-1}$ is corresponding to stretching vibrations of bonds $\mathrm{C}-\mathrm{O}$ from acetate. The FT-IR spectrum of $\mathrm{HP} \beta C D$ showed bands features at $3386,8 \mathrm{~cm}^{-1}$ that may be assign to stretching vibrations of bonds $\mathrm{O}-\mathrm{H}$, at $2970,2 \mathrm{~cm}^{-1}$ and $2931,6 \mathrm{~cm}^{-1}$ due to stretching vibrations of bonds $\mathrm{C}-\mathrm{H}$, at $1643,2 \mathrm{~cm}$ ${ }^{1}$ this is referring to the aldehyde carbonyl group of possible open $\mathrm{CD}$ units.

The FT-IR spectra of physical mixture showed bands features of $\mathrm{HA}$ at $3417,6 \mathrm{~cm}^{-1}$ (for $\mathrm{O}-\mathrm{H}$ stretching vibrations) at $1743,5 \mathrm{~cm}^{-1}$, $1720,4 \mathrm{~cm}^{-1}$ and $1631,7 \mathrm{~cm}^{-1}$ (for $\mathrm{C}=\mathrm{O}$ stretching vibrations), although low in intensity due to high CD concentration are still perceptible in the IV spectrum of the physical mixture. The superposition of absorption spectra of HA and $\mathrm{HP} \beta C D$ was evidenced, indicating the non-inclusion of HA. In the FT-IR spectra of inclusion complex showed no features bands similar to pure HA, the spectrum is very similar to the HP $\beta C D$, proving this manner the $\mathrm{AH}$ inclusion in the HP $\beta C D$ cavity. 
1H-NMR analysis: The Figure 5 shows the $1 \mathrm{H}-\mathrm{RMN}$ from $\mathrm{HP} \beta \mathrm{CD}$ (A) and from the inclusion complex (B). The numbers 1-5 indicate the hydrogen atom signals: from the methyl groups of HP $\beta C D$ (1) and solvent DMSO (2), solvent water (3), H-5 (4), H-3 and H -6 from HP $\beta C D$ (5). Both H-3, H-5 and H-6 protons are located in the inner part of cyclodextrin cavity; H-3 is gravitating towards the wider part of the cone and $\mathrm{H}-5$ towards the narrower part of the cone. ${ }^{18}$. The $1 \mathrm{H}-\mathrm{NMR}$ spectrum of HP $\beta C D(\mathrm{~A})$ shows a signal at $3.62 \mathrm{ppm}$ from $\mathrm{H}-5$ and the other at $3.78 \mathrm{ppm}$ from $\mathrm{H}-3$ and H-6. Inclusion complex (B) shows the H-5 signal noticeably shifted to $3.66 \mathrm{ppm}$ and that of H-3 and H-6 shifted to $3.80 \mathrm{ppm}$. In this way, these shifts from the protons were observed for higher frequency values referring to the hydrogen atoms $\mathrm{H}-3, \mathrm{H}-5$ and $\mathrm{H}-6$ of the $\mathrm{HP} \beta \mathrm{CD}$, which proves the inclusion of HA in HP $\beta C D$.

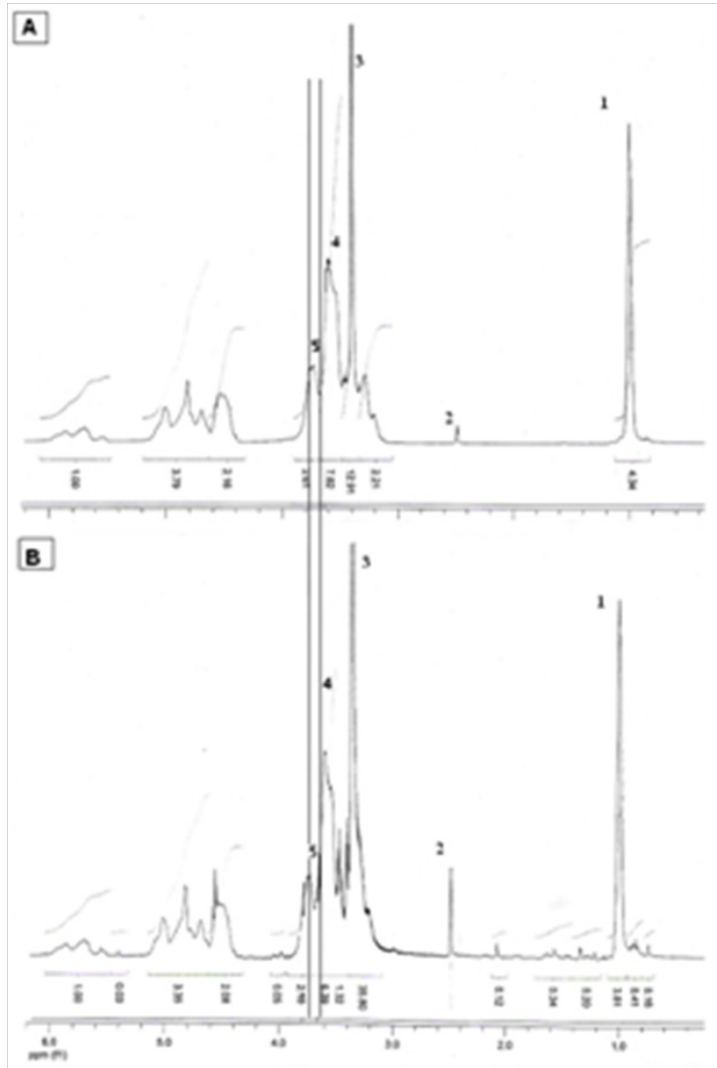

Figure 5 'H-NMR spectra in DMSO-d 6 (solvent) of HP $\beta C D(A)$ and the freeze-dried HA/HP $\beta C D$ inclusion complex (B). The numbers I-5 indicate the signals for the hydrogen atoms of the methyl groups of HPßCD (I), solvent

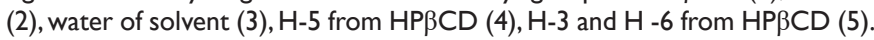

Molecular modeling studies: A model of the inclusion complex was obtained by manual docking of the HA structure within the cavity of the HP $\beta C D$ structure and optimization with the MM+ force field of the HyperChem program. In the optimized model the HA structure remains inside the HP $\beta C D$ cavity, without severe structural deformations.

The conformational changes observed in the HA and HP $\beta C D$ molecules due to the inclusion process were evaluated by the root mean squares (RMS) from the interatomic distances between all the pairs of atoms of each of the molecules before (optimized structures isolated) and after fitting (optimized complex structure). The RMS shift value for HP $\beta C D$ is equal to $1.451 \AA$ and for HA is equal to 0.174 $\AA$. In the case of $\mathrm{HP} \beta C D$ the deviation is greater due to the greater conformational freedom of the side chains of the seven hydroxypropyl groups and in the case of HA, the deviation is much lower, as expected, because the structure of the hormone is very rigid.

The Figure 6 is presented in line model as stick and ball from the inclusion complex, where it is possible to observe the HA molecule inserted in the cavity of the HP $\beta C D$ molecule, and that there is a proximity between the hydrogen atoms $\mathrm{H}-3,-5$ and $\mathrm{H}-6$ of the HP $\beta \mathrm{CD}$ glucose units with the hydrogen atoms of rings $\mathrm{B}, \mathrm{C}$ and $\mathrm{D}$ (Figure 1) of the HA molecule.
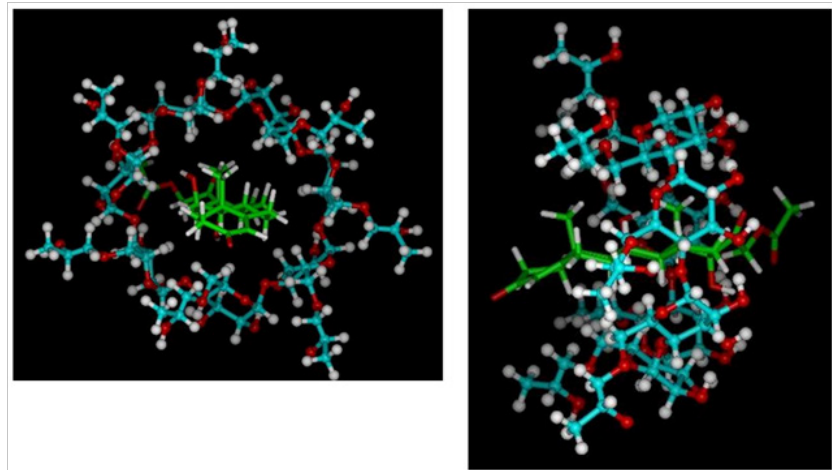

Figure 6 Model of the HA/HP $\beta C D$ inclusion complex obtained by molecular mechanics in stick and ball view mode (frontal and lateral view).

The types of intermolecular interactions involved in the inclusion process are predominantly van der Waals forces. However, the following interactions were observed by hydrogen bonding: one between the oxygen atom of the carbonyl group of acetate of HA and one of the hydroxyls of one of the glucose rings of the $\mathrm{CD}$ that presents distance of $2.91 \AA$ between the respective oxygen atoms and other seven intramolecular interactions, e.g., between hydroxyl groups of adjacent $\mathrm{CD}$ glucose rings.

The Figure 7 (frontal and lateral view) again shows the inclusion complex model in opaque van der Waals solid surface visualization mode HP $\beta C D$ and transparent HA, where it is possible to visualize that the HA completely fills the cavity diameter of the HP $\beta C D$ (front view), and the part of the HA molecule that is included, corresponds to rings $\mathrm{B}, \mathrm{C}$ and $\mathrm{D}$ and which is not fully included, corresponds to the carbonyl groups of ring A and acyl acetate (lateral vision), which are located precisely at the ends of the molecule.
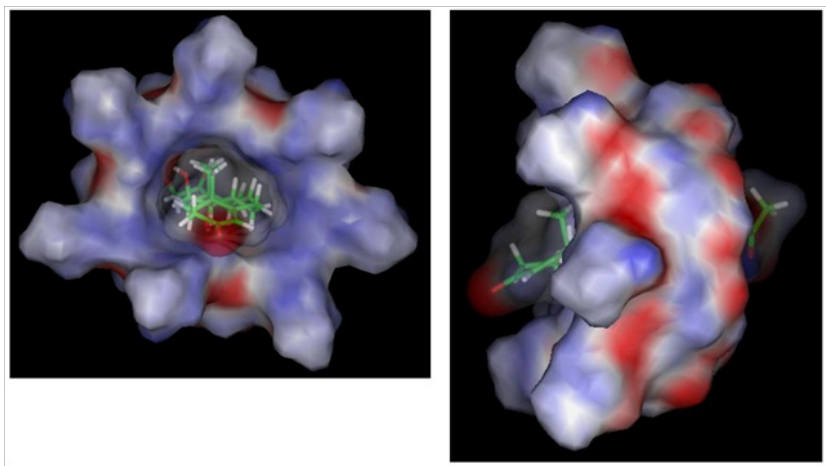

Figure 7 Model of the HA/HP $\beta C D$ inclusion complex obtained by molecular mechanics in stick and ball view mode (frontal and lateral view).

\section{Conclusion}

The development of a new method of inclusion complex of HA with $\mathrm{HP} \beta C D$ allowed the preparation of inclusion complex in a simple and 
efficient manner. The highest percentage of inclusion $(91.52 \pm 0.92 \%)$ was obtained using $5.75 \mathrm{mM}$ HA and $135 \mathrm{mM} H P \beta C D$, water was used as solvent, stirring for 1 hour at the temperature of $37^{\circ} \mathrm{C}$. This percentage of inclusion was higher than that found by Filipović-Grcić J et al. ${ }^{1}$ that was $25 \%$. The AL-type graph and the slope smaller than one indicated a 1:1 stoichiometry. The calculated apparent stability constant $K S=484 \mathrm{M}-1$ of the complex demonstrates an adequate association between HA and the HP $\beta C D$. Results indicated that the solubility property of HA was improved on the order of 64 times by inclusion in the complex HA/HP $\beta C D$ compared with HA crystalline powder (pure). The data obtained from FT-IR and 1H-NMR characterized that an effective HA/HP $\beta C D$ inclusion complex was prepared. Here we have demonstrated that freeze-drying process yield was $77,4 \pm 0,85 \%$. Additionally, molecular modeling studies of the complex at the molar ratio of 1:1 corroborate the experimental data. In the future, the in-vitro dissolution studies will be performed of the inclusion complexion.

\section{Acknowledgments}

The authors would like to thank Cargill (U.S.A.) for the donation of HPßCD. To the Research Center for Natural Products by freezedrying and $1 \mathrm{H}$ NMR. This research was financially supported by CAPES, CNPq and FAPERJ.

\section{Conflicts of interest}

The authors have declared that there is no financial interest or any conflict of interest in the present work.

\section{References}

1. Filipović-Grcić J, Voinovich D, Moneghini M, et al. Chitosan microspheres with hydrocortisone and hydrocortisonehydroxypropyl-b-cyclodextrin inclusion complex. Eur J Pharm Sci. 2000;9(4):373-379.

2. Brazilian Pharmacopoeia. 5a edn, Brasíl. 2017

3. Márcio RMS, Elisabete PS, Rita CSAB, et al. Validation of a New RPHPLC Method for Determination of Hydrocortisone Acetate Complexed with HP $\beta C D$ in an Oral Liquid Pharmaceutical Preparation. J Anal Pharm Res. 2017;6(3):1-5.

4. Giovanna RA, Eleamen A, Silvana CC, et al. Improvement of Solubility and Antifungal Activity of a New Aminothiophene Derivative by Complexation with 2-Hydroxypropyl- $\beta$-cyclodextrin. J Braz Chem Soc. 2017;28(1):116-125.
5. Sunil SJ, Philip B. Cyclodextrins in pharmaceutical formulations II:solubilization, binding constant, and complexation efficiency. Drug Discov Today. 2015;21(2):363-368.

6. Maheshwari A, Saraswat H, Upadhyay SK. Structural insights into the inclusion complexes between clomiphene citrate and $\beta$-cyclodextrin:The mechanism of preferential isomeric selection. Chirality. 2017;29(8):451457 .

7. Zingone G, Rubessa F. Preformulation study of the inclusion complex warfarin-beta-cyclodextrin. Int J Pharm. 2005;291(4):3-10.

8. Jing W, Yanping $\mathrm{C}$, Baoguo $\mathrm{S}$, et al. Physicochemical and release characterisation of garlic oil- $\beta$-cyclodextrin inclusion complexes. Food Chemistry. 2011;127(4):1680-1685.

9. Rui Z, Corine S, Haiyang Z, et al. NMR Study on the Inclusion Complexes of $\beta$-Cyclodextrin with Isoflavones. Molecules. 2016;21(372):1-11.

10. Sanjoy KD, Rajan R, Sheba D, et al. Cyclodextrins - The Molecular Container. Res J Pharm Biol Chem Sci. 2013;4(2):1694-1720.

11. Tijana M, Kyriakos K, Adrijana G, et al. Improvement of Aripiprazole Solubility by Complexation with (2-Hydroxy) propyl- $\beta$-cyclodextrin Using Spray Drying Technique. AAPS PharmSciTech. 2012;13(2):623631

12. Morillo E, Sánchez-Trujillo MA, Villaverde J, et al. Effect of contact time and the use of hydroxypropyl- $\beta$-cyclodextrin in the removal of fluorene and fluoranthene from contaminated soils. Science of the Total Environment. 2014;496:144-154.

13. Higuchi T, Connors KA. Phase Solubility Techniques. Adv Anal Chem Instrum. 1965;4:117.

14. Jing L, Qihua J, Ping D, et al. The formation of a host-guest inclusion complex system between b-cyclodextrin and baicalin and its dissolution characteristics. J Pharm Pharmacol. 2017;69(6):663-674.

15. Lebrilla CB. The Gas-Phase Chemistry of Cyclodextrin Inclusion Complexes. AccChem Res. 2001;34(8):653-661.

16. Lipkowitz KB. Applications of computational chemistry to the study of cyclodextrins. Chem Rev 1998;98(5):1829-1874.

17. Benjamas C, Jaruporn R. Inclusion complex formation of cyclodextrin with its guest and their applications. Biol Eng Med. 2016;2(1):1-6.

18. Vesna N, Mihajlo S, Ljubis N, et al. Inclusion complexes with cyclodextrin and usnic acid. J Incl Phenom Macrocycl Chem. 2013;76(1-2):173-182. 